"This document is the Accepted Manuscript version of a Published Work that appeared in final form in ACS Nano 2017, 11, 9321-9329, copyright (C) American Chemical Society after peer review and technical editing by the publisher. To access the final edited and published work see 10.1021/acsnano.7b04728"

\title{
Nonacene generated by on-surface dehydrogenation
}

\author{
Rafal Zuzak ${ }^{1}$, Ruth Dorel ${ }^{2}$, Mariusz Krawiec ${ }^{3}$, Bartosz Such ${ }^{1}$, Marek Kolmer ${ }^{1}$, Marek \\ Szymonski ${ }^{1}$, Antonio M. Echavarren ${ }^{2,4}$, Szymon Godlewski ${ }^{1{ }^{1, *}}$ \\ ${ }^{1}$ Centre for Nanometer-Scale Science and Advanced Materials, NANOSAM, Faculty of Physics, \\ Astronomy and Applied Computer Science, Jagiellonian University, Łojasiewicza 11, PL 30-348 \\ Krakow, Poland \\ 2 Institute of Chemical Research of Catalonia (ICIQ), Barcelona Institute of Science and \\ Technology, Av. Països Catalans 16, 43007 Tarragona, Spain \\ ${ }^{3}$ Institute of Physics, M. Curie-Sklodowska University, P1. M. Curie-Skłodowskiej 1, 20-031 \\ Lublin, Poland \\ ${ }^{4}$ Departament de Química Orgànica i Analítica, Universitat Rovira i Virgili, C/ Marcel·lí \\ Domingo s/n, 43007 Tarragona, Spain \\ "e-mail: szymon.godlewski@uj.edu.pl
}

The on-surface synthesis of nonacene has been accomplished by dehydrogenation of an air-stable partially saturated precursor, which could be aromatized by using a combined scanning tunnelling and atomic force microscope (STM/AFM) tip as well as by on-surface annealing. This transformation allowed the in-detail analysis of the electronic properties of nonacene molecules physisorbed on $\mathrm{Au}(111)$ by scanning tunnelling spectroscopy (STS) measurements, which were corroborated by density theory functional (DFT) calculations thus confirming the spatial mapping of its molecular orbitals. Furthermore, the thermally-induced dehydrogenation uncovered the isomerisation of intermediate dihydrononacene species, which allowed for their in-depth structural and electronic characterization.

Acenes are an important class of polycyclic aromatic hydrocarbons constituted by linearly fused benzene rings and are considered to be central to the development of the future generation of (opto)electronic devices. These molecules have been the subject of extensive study due to their unique electronic and magnetic properties ${ }^{1-5}$, which make them promising candidates to be implemented as low bandgap semiconducting materials in molecular electronic devices such as field-effect transistors ${ }^{6}$, photovoltaic devices ${ }^{7}$, or light emitting diodes ${ }^{8}$. The electronic properties of acenes have been predicted to be improved for higher acenes anticipating narrower HOMOLUMO (highest occupied molecular orbital - lowest unoccupied molecular orbital) gaps as well as lower reorganization energies as the number of annealed rings grows ${ }^{9-12}$. At the same time, acenes could be regarded as the narrowest graphene nanoribbons (GNR) with zig-zag edge 
topology suggesting potential applicability in spintronics ${ }^{13}$ and plasmonics ${ }^{14}$. Nevertheless, both the preparation and the fundamental study of extended acenes are limited by their intrinsic instability and low solubility, which has prompted the development of a variety of synthetic methods and stabilization strategies that allow gaining access to acenes longer than pentacene ${ }^{15-}$ 19

Despite initial reports dating from the $1940 \mathrm{~s}$ and $1950 \mathrm{~s}^{20-21}$, it was not until recently that the Strating-Zwanenburg reaction of stable $\alpha$-(diketo)precursors combined with low-temperature matrix-isolation techniques allowed the first unambiguous generation and spectroscopic characterization of hexacene ${ }^{22}$ and heptacene ${ }^{23}$, which could also later be synthesized and isolated in bulk in the solid state ${ }^{24-26}$. Octacene and nonacene were likewise generated through the photoinduced decarbonylation of the corresponding bis( $\alpha$-diketo)precursors in a cryogenic argon matrix ${ }^{27}$, which constitutes the sole evidence for the existence of nonacene reported to date. However, despite kinetically stabilized nonacene derivatives have been successfully isolated ${ }^{28-29}$, the detailed electronic structure of the parent nonacene has not yet been established.

In recent years, on-surface synthesis has attracted much attention from the scientific community, offering a breakthrough in the atomically precise assembly of molecular nano-architectures ${ }^{30-32}$. The enormous progress in scanning probe techniques has boosted the planar molecule manipulation and tip-induced chemical reactions to an unprecedented level of precision ${ }^{33-39}$. Moreover, the combination of ultra-high vacuum conditions with cryogenic temperatures and application of chemically inert substrates has also made feasible the synthesis and detailed characterization of molecules otherwise too reactive and therefore unstable under ambient conditions $^{40-41}$. The ability of tip functionalized non-contact atomic force microscopy (NC-AFM) to resolve chemical structures ${ }^{42-46}$ is complementary to the scanning tunnelling microscopy/spectroscopy (STM/STS) electronic orbital imaging ${ }^{47-50}$ toward detailed and complete molecule description. In the last two years the on-surface chemistry approach has also been applied for the generation of middle-sized acenes such as tetracene ${ }^{51}$ and hexacene ${ }^{52}$ by deoxygenation of stable molecular precursors. We have recently developed a method for the preparation of hydroacenes, which can be considered as stable 'hydrogen protected acenes, ${ }^{53}$, based on the gold(I)-catalyzed cyclization of aryl-tethered 1,7-enynes ${ }^{54}$. Thus, we envisioned that the hydroacenes accessed through our method could be suitable precursors for the generation of otherwise elusive higher acenes.

Herein we present the on-surface generation of nonacene as well as the detailed study of its electronic structure on a $\mathrm{Au}(111)$ surface. Our method is based on the atomically precise step-bystep dehydrogenation of a stable and easily handled tetrahydrononacene precursor $\mathbf{N n}-\mathbf{4 H}$ $(6,10,17,21 \text {-tetrahydrononacene })^{54}$ with the application of the tip of combined STM/AFM setup (Scheme 1a). High resolution NC-AFM imaging is applied for the detailed visualization of the 
internal structure of generated long acenes. Details on the electronic structure of the generated nonacene molecules, as well as the intermediate 6,21-dihydrononacene (Nn-2H-3), are revealed using high resolution $\mathrm{dI} / \mathrm{dV}$ mapping, which allows to visualize spatially the electron density cloud. Our measurements showed that not only frontier molecular orbitals (i.e. HOMO and LUMO), but also deeper lying ones (i.e. HOMO-1 and LUMO+1) could be imaged with submolecular resolution. Additionally, we demonstrated that the surface assisted dehydrogenation process that leads to the generation of nonacene could also be induced thermally, constituting a highly efficient method for the preparation of increased amounts of this long acene. We observed that in this case the hydrogen abstraction process was accompanied by a series of 1,3-hydrogen shifts over the dihydrononacene species resulting in a variety of $\mathbf{N n - 2 H}$ isomers with the nonaromatic ring located at different positions within the internal structure of the molecule, which therefore allowed the electronic characterization of a range of $\mathbf{N n - 2 H}$ isomers (Scheme 1b).

a)

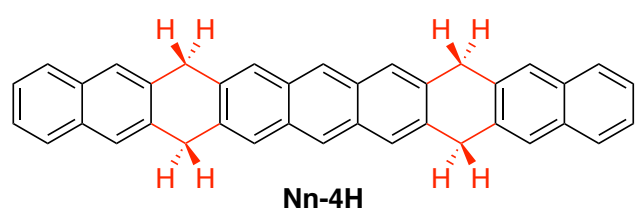

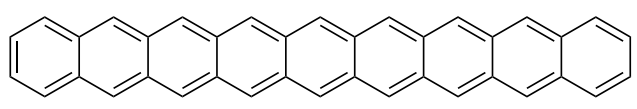

nonacene b)<smiles>NCC[C@@H]1[C@@H]2[CH]c3cc4cc5cc6cc7cc8ccccc8cc7cc6cc5cc4cc3[C@@H]1c1cc3ccccc3cc12</smiles><smiles></smiles>

$\mathrm{Nn}-2 \mathrm{H}-4$

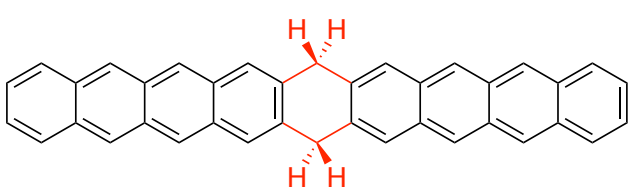

$\mathrm{Nn}-2 \mathrm{H}-5$

Scheme 1. On-surface synthesis of nonacene; (a) Nonacene generated from 6,10,17,21-tetrahydononacene (Nn$4 \mathrm{H})$ precursor; (b) Dihydrononacene intermediates $(\mathrm{Nn}-2 \mathrm{H})$ observed on $\mathrm{Au}(111)$.

\section{Results}

Tip-induced on-surface generation of nonacene. For the experiments reported here we sublimed molecules on the $\mathrm{Au}(111)$ surface at room temperature. The starting material for our research was $\mathbf{N n - 4 H}$, a molecule with two non-aromatic rings bearing each two methylene groups susceptible of being dehydrogenated ${ }^{54}$. After deposition, the molecules preferentially physisorbed at the corners of the herringbone surface (red contours in Figure 1a,b) patterning similarly to pentacene molecules, as reported by Soe et $a l^{48}$. When the amount of molecules increased they tended to follow the herringbone reconstruction pattern as shown in Figure 1a,b. However, the molecules located at reconstruction elbows were ultimately more stable and were therefore used for the detailed characterization in this study. The presence of the two non-aromatic rings was discernible in filled- and especially empty-state STM topographies (Figure 1c), as well as in the 
high resolution constant height NC-AFM image (Figure 1d), proving that the thermal evaporation of the material does not lead in general to molecule defragmentation. Nevertheless, we noted that a small fraction of molecules underwent hydrogen abstraction spontaneously and were found as Nn-2H-3, marked by white contours in Figure 1b. A deeper analysis of the STM appearance of Nn-4H indicated that the enhanced contrast over the methylene moieties in the non-aromatic rings was recorded for voltages exceeding $+1.95 \mathrm{~V}$. This correlates with the STS resonance recorded over the non-aromatic rings pointing to the increased empty state density cloud (see Supplementary Information Figure S4). The presence of the characteristic large lobes located above the non-aromatic rings in the empty state STM images provided a hint to discriminate the planar and aromatic board of molecules fabricated by atom manipulation from the non-planar starting molecules. Tip-induced cleavage of one of the $\mathrm{C}_{\mathrm{sp} 3}-\mathrm{H}$ bonds within the non-aromatic ring occurred when the apex was located over the methylene selected for manipulation and the tunneling current was increased to several hundreds of $\mathrm{pA}$ with the feedback loop turned off. The sudden drop of the current was a signature of hydrogen abstraction (see Supplementary Information Figure S5). We found in our experiments that within the time resolution of the STM/AFM apparatus two hydrogen atoms of the initially non-aromatic ring, one from each methylene moiety, were extracted leading to its aromatization. The application of the STM/AFM setup did not allow to determine the details of the abstraction reaction and consequently did not allow to discern whether both hydrogen atoms were abstracted in a concerted or a stepwise process. Nonetheless, the straight removal of the two hydrogen atoms associated with planarization and aromatization of the ring was not surprising as the cleavage of one of the benzylic $\mathrm{C}-\mathrm{H}$ bonds would result in a highly reactive radical intermediate that should rapidly lead to aromatization upon loss of a second hydrogen atom. In our experiments, we noted that the dehydrogenation of $\mathbf{N n - 4 H}$ only occurred for voltages higher than $+1.95 \mathrm{~V}$. This threshold corresponds to the resonant tunneling through the recorded state (see Supplementary Information Figure S4) and is much lower than the $\mathrm{C}-\mathrm{H}$ bond dissociation energy ${ }^{39,55}$, which indicates that the energy dissipated from vibronic excitations cleaves the bonds. We note here that the $\mathrm{C}_{\mathrm{sp} 3}-\mathrm{H}$ bonds in the methylene groups are the anticipated breaking points, since their bond-dissociation energy (3.4 eV for 9,10-dihydroanthracene $\mathrm{e}^{39,55}$ ) is lower compared to $\mathrm{C}_{\mathrm{sp} 2}-\mathrm{H}$ bonds in the aromatic rings (e.g. $4.8 \mathrm{eV}$ for benzene ${ }^{39,56}$ ). The observed reaction is reminiscent to the recently reported C-H bond cleavage that leads to triangulene ${ }^{40}$. The STM appearance of the intermediate $\mathbf{N n - 2 H - 3}$ generated using the above described approach is shown in Figure 1e. Upon aromatization of one of the initially non-aromatic rings we clearly noticed the enhancement of the intramolecular ladder contrast characteristic for the acene filled-state images and the disappearance of the pronounced empty-state lobes associated with the presence of the methylene groups in the starting material. Importantly, the remaining two $\mathrm{CH}_{2}$ groups exhibit the characteristic empty-state twoside-lobe appearance at significantly lower voltages compared to $\mathbf{N n}-\mathbf{4 H}$, i.e. starting from +1.2 
V, which correlates with the STS measured resonance (see Supplementary Information Figure S1) resulting from extension of the central aromatic backbone of the molecule to a hexacene-like moiety upon aromatization. This is even better visualized in NC-AFM images, shown in Figure $1 \mathrm{f}$, in which the six fused benzene rings are clearly discernible. The final target nonacene molecule was fabricated by application of the same dehydrogenation procedure to the second nonaromatic ring. However, we found an important difference in the process responsible for the transformation of Nn-2H-3 into nonacene, compared to the first dehydrogenation of Nn-4H into Nn-2H-3. The efficient dehydrogenation was already recorded at voltages starting from $+1.2 \mathrm{~V}$, which correlates with the significantly lower energy of the delocalized LUMO orbital of $\mathbf{N n - 2 H}-$ 3 (recorded in STS at $1.12 \mathrm{~V}$, see Supplementary Information Figure S1) compared with the energy of Nn-4H LUMO orbital. This finding further corroborates the extraction mechanism based on vibronic excitation. The filled state STM image (Figure 1g) of the final product nonacene - clearly shows the ladder internal contrast with the expected nine lobes along the molecule, whereas in the empty state image the bright lobes related to the presence of $\mathrm{CH}_{2}$ groups are obviously absent. High-resolution NC-AFM image displayed in Figure 1h clearly shows presence of linearly fused nine benzene rings, doubtlessly proving generation of the nonacene molecule. 
a

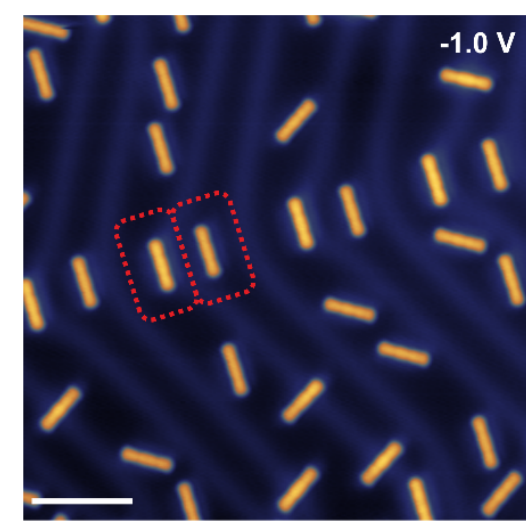

b

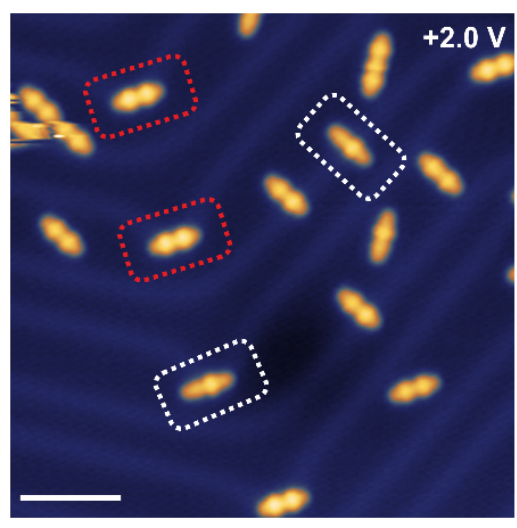

C
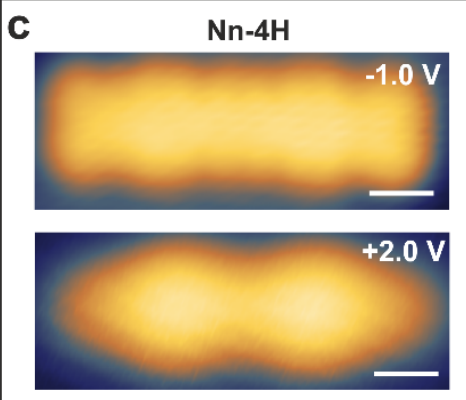

\section{e}

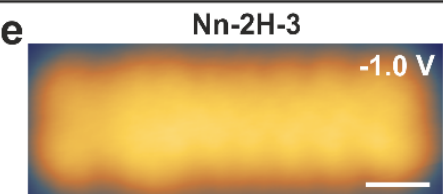

$+2.0 \mathrm{~V}$
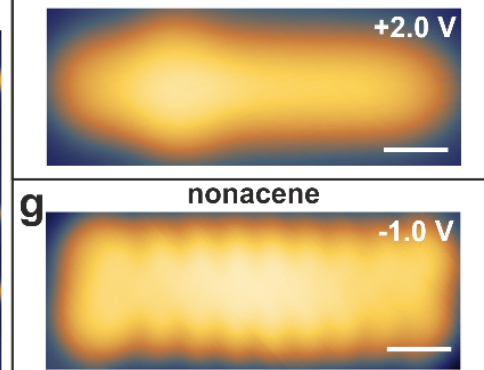

$+2.0 \mathrm{~V}$
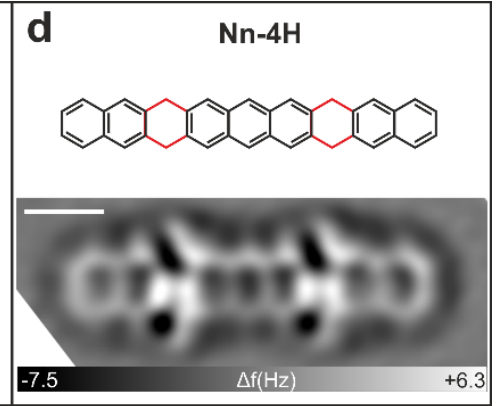

f

$\mathrm{Nn}-2 \mathrm{H}-3$
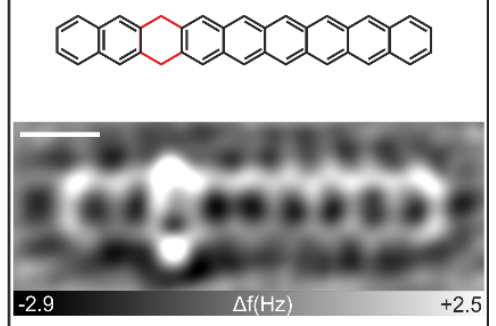

h

nonacene
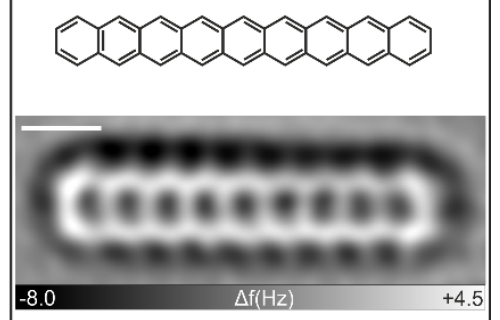

Figure 1. (a) Filled and (b) empty state STM topographies of $\mathrm{Au}(111)$ sample after $\mathrm{Nn}-4 \mathrm{H}$ deposition. Red-dashed contours mark $\mathrm{Nn}-4 \mathrm{H}$ physisorbed at herringbone reconstruction elbows, white-dashed contours indicate molecules that spontaneously underwent dehydrogenation into $\mathrm{Nn}-2 \mathrm{H}-3$. High resolution empty- and filled-state STM constant current topographies with corresponding Laplace filtered NC-AFM constant height images of Nn-4H $(c, d), N n-2 H-3(e, f)$ and nonacene $(g, h)$. Molecules shown in $(e, f)$ and $(g, h)$ were generated by tip-induced dehydrogenation; tunnelling current: 50 pA (a), 30 pA (b), 100 pA (c, upper image), 25 pA (c, lower image), 35 pA (e), $50 \mathrm{pA}$ (g, upper image), $45 \mathrm{pA}$ (g, lower image); NC-AFM oscillation amplitude: $113 \mathrm{pm}$ (d,f), $57 \mathrm{pm}(\mathrm{h})$.

Nonacene electronic properties. In order to confirm the on-surface generation of nonacene and to provide its detailed electronic structure characterization we performed STS measurements and $\mathrm{dI} / \mathrm{dV}$ mapping of its molecular orbitals using a lock-in amplifier. For STS measurements only tips allowing for clear identification of the $\mathrm{Au}(111)$ surface state resonance at approximately -0.5 V were used. Taking into account that the electron cloud is spatially distributed over the molecule and in order to avoid measurements at positions that correspond to nodal planes of monoelectronic orbitals, which would lead to suppression of the dI/dV STS signal corresponding to these orbitals, we performed point STS measurements at several different horizontal locations of the tip apex. Based on previous reports for shorter acenes, namely pentacene $e^{47-48}$ and hexacene $e^{52}$, we expected the most prominent signal to be recorded at the corners of the molecule (marked by a circle in Figure 2a left inset) for filled-states spectroscopy and at the ends (indicated by a circle in Figure 2a right inset) for empty states measurements. Therefore, for point spectroscopy measurements we positioned the tip in the above mentioned different locations, as indicated by blue dots in the insets in Figure 2a. The recorded differential conductance spectra contain strong filled-state 
resonances at $-1.00 \mathrm{~V}$ and $-0.34 \mathrm{~V}$ and slightly broader resonances in the empty state regime at $+0.85 \mathrm{~V}$ and $+1.70 \mathrm{~V}$. Additionally, a plain shoulder can also be noticed positioned within filled states at around $-1.65 \mathrm{~V}$. In order to determine if the recorded peaks could be attributed to the measurements of the molecular orbitals in a mono-electronic approximation we performed spatial $\mathrm{d} / \mathrm{dV}$ mapping at the voltages corresponding to these resonances and compared those results with the simulated $\mathrm{d} / \mathrm{dV}$ maps obtained on the basis of the simple Tersoff-Hamann approximation (Figure 2b-e) $)^{57}$. The calculations were performed for the gas phase molecule configuration due to the weak interaction of the molecule with the substrate and expected insignificant influence on the orbital distribution. The maps recorded at $-1.00 \mathrm{~V}$ and $-0.34 \mathrm{~V}$ showed two rows containing 8 and 9 lobes, respectively, and a nodal plane along the molecule. These maps correspond to the spatial distribution of the HOMO-1 and HOMO electronic orbitals of the free nonacene molecule as corroborated by the simulated images. This is in line with the expected single electron wave function signature of the linearly fused benzene rings, for which it is expected to record $2 n(2 n$ 2) lobes separated by a nodal plane along the molecule backbone for HOMO (HOMO-1) of the $n$-acene molecule. This allowed us to unambiguously assign the peaks recorded at $-1.00 \mathrm{~V}$ and $0.34 \mathrm{~V}$ as the signature of HOMO-1 (Figure 2b) and HOMO (Figure 2c) single electron orbitals, respectively. We did not map the spatial distribution of the $\mathrm{dI} / \mathrm{dV}$ signal at the voltage corresponding to the lowest lying peak recorded at $-1.65 \mathrm{~V}$, which for consistency, we denote as HOMO-2, although in previous reports for shorter acenes it became clear that the recorded $\mathrm{dI} / \mathrm{dV}$ maps largely deviated from expected for spatial mapping of purely mono-electronic HOMO-2 orbital for pentacene ${ }^{48}$ and hexacene ${ }^{52}$. Comparison of the $\mathrm{dI} / \mathrm{dV}$ maps recorded at voltages corresponding to the peaks at $+0.85 \mathrm{~V}$ and $+1.70 \mathrm{~V}$ with the ones simulated for LUMO (Figure 2d) and LUMO+1 (Figure 2e) monoelectronic wavefunctions showed striking agreement thus confirming the assignment of these peaks. The only minor discrepancy for both maps was the experimental missing of the faintest, banana-shape features located in the closest proximity to the large, oval end-lobes. This is, however, in agreement with the results obtained for pentacene and hexacene physisorbed on $\mathrm{Au}(111)$ and assigned to limited spatial tip resolution ${ }^{52}$. Despite it has been theoretically predicted ${ }^{58-61}$ and experimentally evidenced ${ }^{27}$ that nonacene might be characterized by an antiferromagnetic ground state structure with open-shell singlet state, it is important to note that we have obtained the excellent agreement between computed and experimental $\mathrm{dI} / \mathrm{dV}$ maps for the non-magnetic structure of nonacene corresponding to the closedshell ground state configuration. This can be easily rationalized taking into account that it is expected for the magnetic anisotropy to be suppressed for molecules interacting with a $\mathrm{Au}(111)$ surface. Hence, we conclude that for the longest acene known to date, namely nonacene, we have successfully mapped spatially not only the frontier molecular orbitals, i.e. HOMO and LUMO, but also deeper lying HOMO-1 and LUMO+1 ones. 


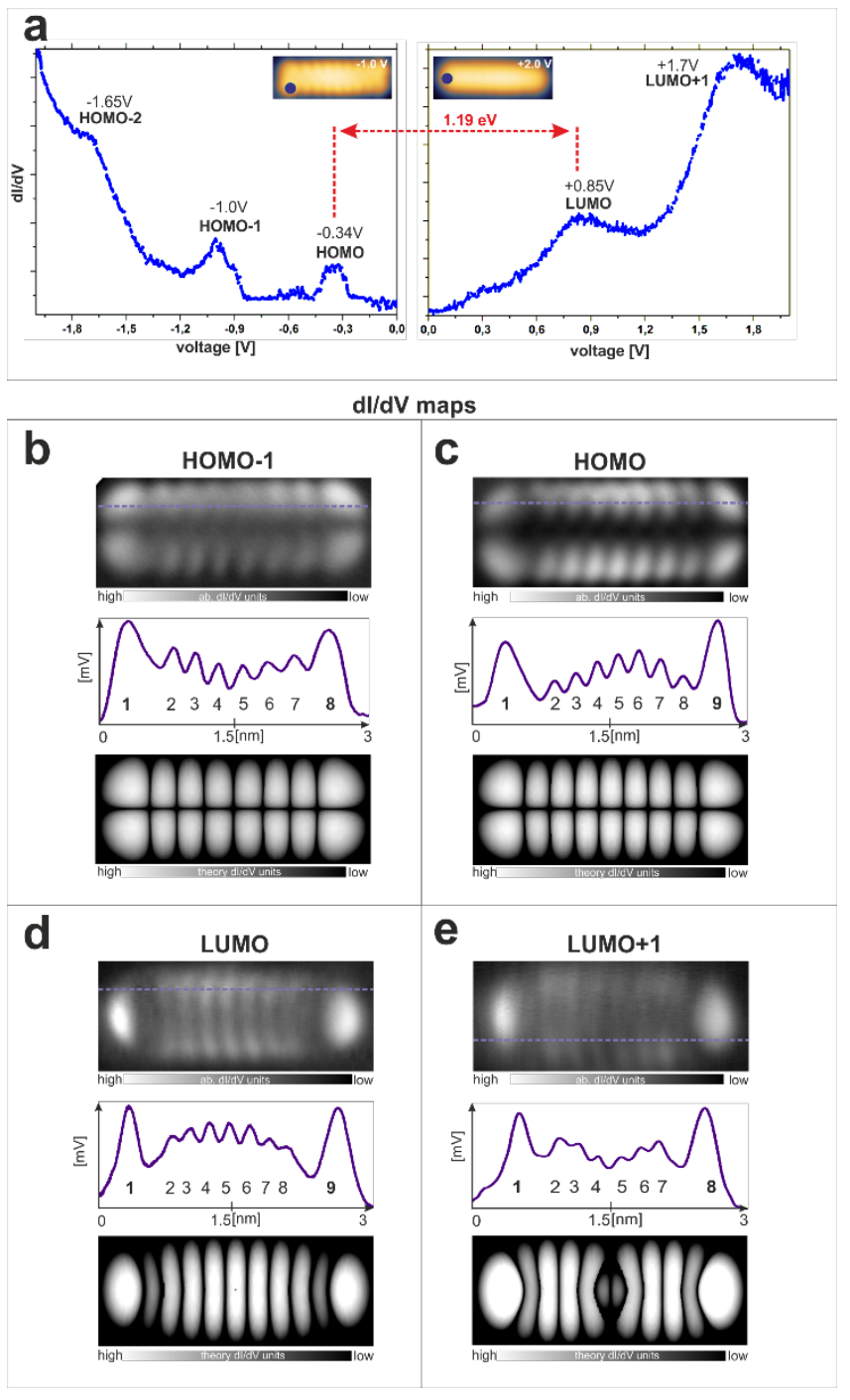

Figure 2. (a) Single point STS data recorded over a nonacene molecule. Insets show the lateral tip position during filled (left panel, $100 \mathrm{pA}$ ) and empty (right panel, $50 \mathrm{pA}$ ) state spectroscopy data acquisition. (b-e) Experimental $\mathrm{dl} / \mathrm{dV}$ spatial maps recorded at energies corresponding to resonances shown in (a) complemented by theoretically calculated maps, cross-sections along experimental maps are displayed to guide the eye in symmetry analysis; tunnelling current: $100 \mathrm{pA}(\mathrm{b}, \mathrm{c}), 50 \mathrm{pA}(\mathrm{d}, \mathrm{e})$.

Having assigned the STS recorded resonances, we infer that the STS recorded transport gap of nonacene equals approximately $1.2 \mathrm{eV}$, which follows the expected reduction of gap with increased length of the fused benzene rings system and is in good agreement with the optical gap determination of photochemically-generated nonacene, which needed to be performed by irradiation in a solid Ar matrix ${ }^{27}$ and in which, from the UV-Vis spectrum, a HOMO-LUMO gap of $1.4 \mathrm{eV}$ could be estimated.

Thermally-induced on-surface generation of nonacene. The above reported procedure for the on-surface generation of nonacene based on atomic manipulation is very precise, although not efficient for the preparation of increased amounts of molecules of this long acene. Therefore, we decided to adapt an alternative approach based on the surface-assisted thermally-induced dehydrogenation of $\mathbf{N n}-\mathbf{4 H}$, which would allow for the efficient parallel preparation of a whole 
array of nonacene species by annealing after molecule deposition. The empty state STM image recorded for the sample heated at $150{ }^{\circ} \mathrm{C}$ clearly indicated that the majority of $\mathbf{N n}-\mathbf{4 H}$ molecules underwent a transition (Figure 3a). Hence, whereas a small fraction of them stayed intact and exhibited the characteristic STM appearance for the starting material with 4 pronounced lobes (see the molecules marked by red dashed contours in Figure 3a), the vast majority was found as Nn-2H species with clearly visible markers of the two remaining $\mathrm{CH}_{2}$ groups. However, a detailed inspection revealed that the dihydrononacenes present on the surface were not identical since the bright lobe corresponding to the non-aromatic ring was found at different positions. This is documented in high resolution STM images shown in Figure 3c-e, which present the different Nn-2H isomers obtained after thermal treatment. We found that the most abundant isomer was the symmetric 8,19-dihydrononacene (Nn-2H-5) (Figure 3e). The sample contained also 7,20dihydrononacenes (Nn-2H-4, Figure 3d) and already described in the first section Nn-2H-3 (Figure 3c), the latter being formed directly from $\mathbf{N n}-\mathbf{4 H}$ by hydrogen removal without subsequent hydrogen migration. Our experiments demonstrated that upon dehydrogenation of the starting material, which leads to $\mathbf{N n - 2 H - 3 , ~ t h e ~ h y d r o g e n ~ a t o m s ~ i n ~ t h e ~ r e m a i n i n g ~ m e t h y l e n e ~}$ moieties migrate along the molecule with a preference to be located within the central ring. The overall transformation is similar to the thermally-induced 1,3-hydrogen migration sequence reported for the conversion of 5,14-dihydropentacene into thermodynamically more stable 6,13dihydropentacene $^{62}$. Furthermore, this transformation is in agreement with our gas phase DFT calculations, which show that the Nn-2H-4 and Nn-2H-5 are characterized by an energy lowering of $85 \mathrm{meV}$ and $100 \mathrm{meV}$, respectively, with respect to $\mathbf{N n - 2 H - 3}$. 
a

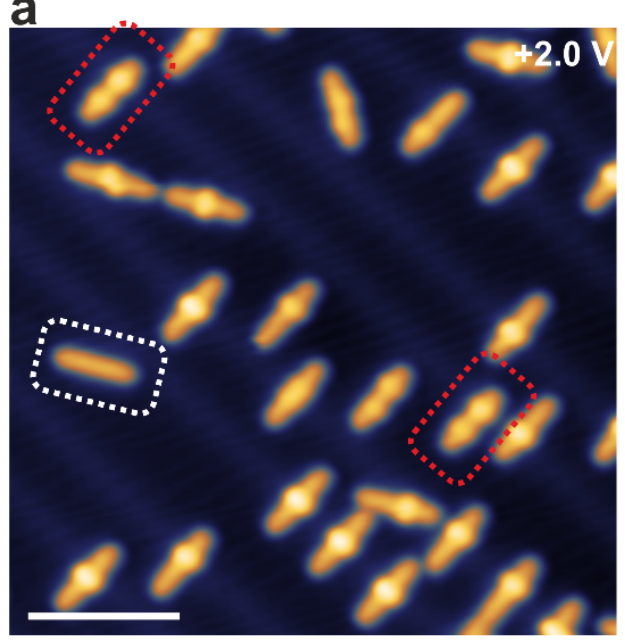

C
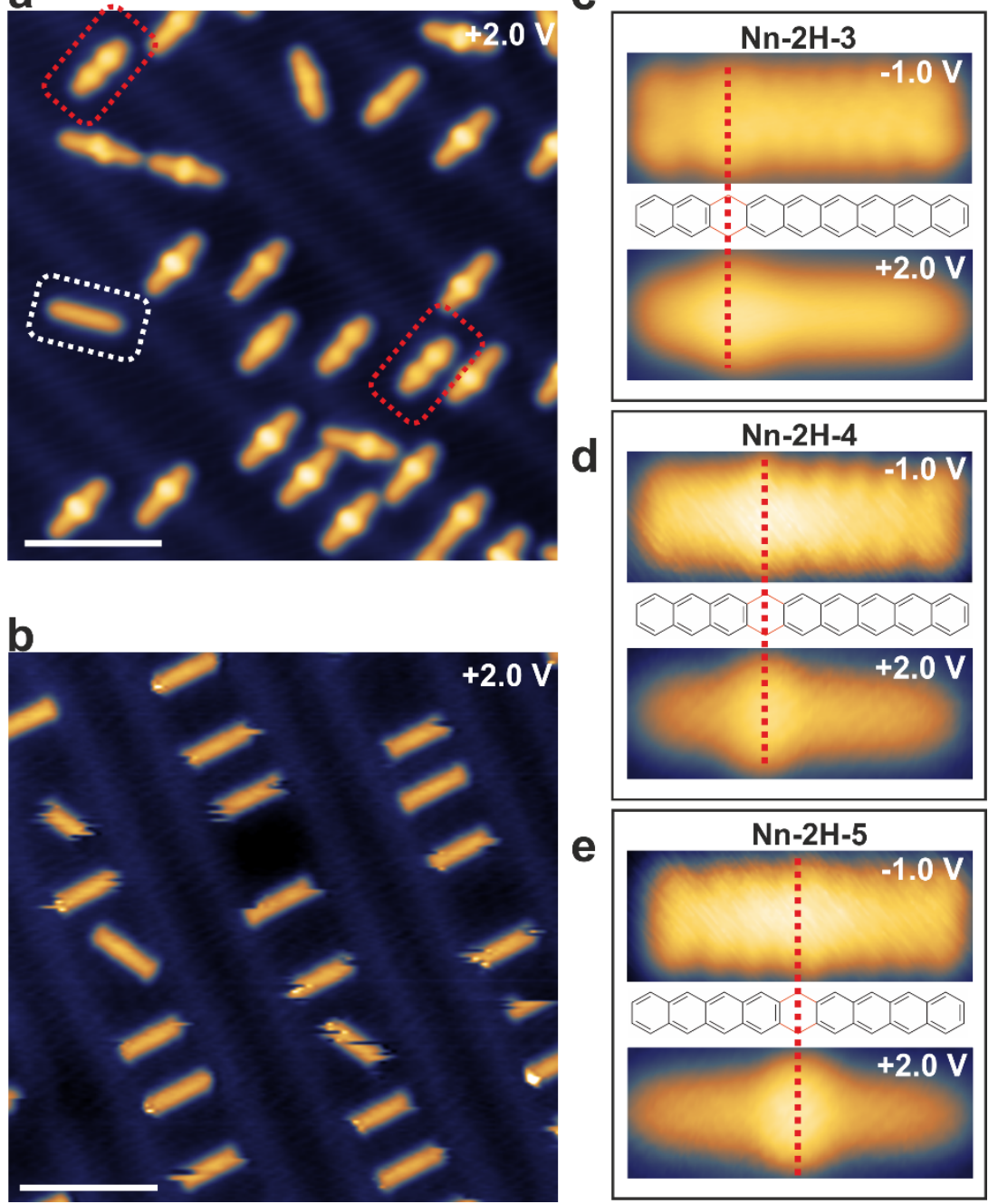

d

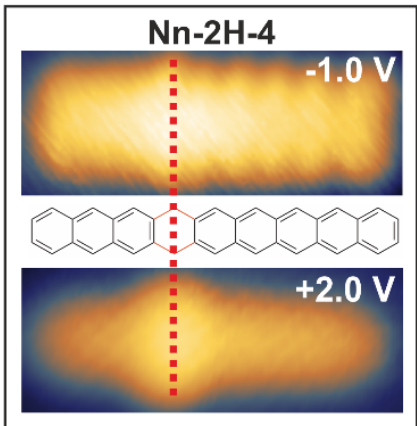

e

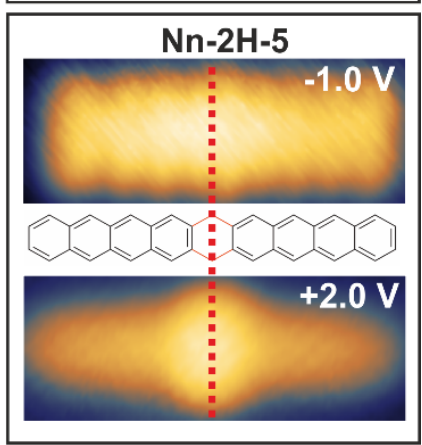

Figure 3. Empty state STM topographies showing the sample after annealing to $150^{\circ} \mathrm{C}$ (a) and $210^{\circ} \mathrm{C}$ (b), the white contour marks the nonacene molecule, whereas the red ones point the $\mathrm{Nn}-4 \mathrm{H}$. The streaky pattern clearly discernible above molecules in panel (b) results from high mobility of nonacene species, which are not located in the herringbone reconstruction elbows; (c), (d) and (e) high resolution filled and empty state STM images of different $\mathrm{Nn}-2 \mathrm{H}$ isomers generated by thermal hydrogen removal and on-surface isomerisation; red dashed line indicates the position of the non-aromatic ring, tunnelling current: 30 pA (a,b), 35 pA (c), 100 pA (d, upper image), $50 \mathrm{pA}$ (d, lower image), $100 \mathrm{pA}$ (e, upper image), 30 pA (e, lower image).

Our experiments showed that thermal treatment at a higher temperature of $210{ }^{\circ} \mathrm{C}$ results in the complete dehydrogenation of all the starting $\mathbf{N n}-\mathbf{4 H}$ species leading to an on-surface generated array of well separated fully aromatic nonacene molecules, as documented in Figure 3b.

\section{Discussion}

Our results demonstrate that partially saturated acene derivatives - hydroacenes - are indeed 'hydrogen protected' acenes and can therefore be used as suitable precursors for the synthesis of the corresponding parent acenes. We have successfully generated nonacene on the $\mathrm{Au}(111)$ surface by atomically precise step-by-step hydrogen desorption performed with the application of combined STM/AFM setup. The detailed analysis of the hydrogen abstraction process revealed that it is efficiently initiated when the STM tip is placed over the methylene groups and the bias voltage is raised above the value corresponding to the LUMO energy. Importantly, the threshold 
voltage was found to differ between dihydrononacene and tetrahydronoancene species, which follows the decrease of the LUMO orbital energy associated to an extended aromatic backbone. This demonstrates that the desorption occurs during resonant tunneling, which indicates on the vibronic mechanism of hydrogen removal. Furthermore, we have applied point STS measurements and $\mathrm{dI} / \mathrm{dV}$ mapping to record spatial distribution of frontier, i.e. HOMO and LUMO, as well as deeper lying HOMO-1, and LUMO+1 molecular orbitals. Spectroscopic measurements revealed that the STS recorded transport gap reaches $1.19 \mathrm{eV}$, which is in reasonable agreement with optical gap measurements reported by Tönshoff et $a l^{27}$.

We have proven that annealing of the sample provides an efficient approach to generate a larger amount of nonacene molecules. Additionally, we have found that the thermally induced dehydrogenation is associated with isomerisation of the dihydrononacene intermediates by migration of hydrogen atoms located in methylene moieties toward the central ring of the molecule. The generation of different $\mathbf{N n - 2 H}$ isomers boosted additional experiments toward the detailed analysis of their electronic properties. The non-aromatic ring divides the backbone of the species into two aromatic platforms. By STS measurements complemented with $\mathrm{dI} / \mathrm{dV}$ spatial mapping we found that the electronic properties are determined by the orbitals, which are separately localized over the aromatic skeletons. In other words, e.g., HOMO (LUMO) orbital of Nn-2H-4 and Nn-2H-3 is spatially extended only over the pentacene or hexacene moiety of the dihydrononacene, respectively (see Supplementary Information Figure S2 and S1). The STS resonances recorded over the shorter aromatic platforms, i.e., anthracene and naphthalene moieties, are found at much higher energies as expected for short acenes. The detailed analysis revealed that the energies of HOMO and LUMO resonances of Nn-2H-3, which correspond to $0.65 \mathrm{~V}$ and $+1.25 \mathrm{~V}$, respectively, perfectly match the values from STS measurements for hexacene on $\mathrm{Au}(111)$ reported by Krüger et $a l^{52}$. Similarly, the data obtained for Nn-2H-4 are in good agreement with the resonances recorded for pentacene by Soe $e t \mathrm{al}^{48}$. These findings suggest that the frontier electronic levels delocalized over the longest aromatic moiety are not impaired significantly by the presence of the second aromatic backbone separated by the non-aromatic ring. However, the spatial distribution of the electronic cloud is modified at internal peripheries of the aromatic cores (see Supplementary Information Figure S1-S3). In particular, by analyzing the projected density of states, we found that the projection of delocalized orbitals at the states of hydrogen atoms within the methylene groups exhibits an important contribution. This finding explains the appearance of pronounced lobes associated with the presence of non-aromatic rings in STM topographies of dihydro- and tetrahydrononacene molecules.

In summary, our experiments demonstrated that the on-surface generation of nonacene can be achieved by dehydrogenation of partially saturated molecular precursors. This opens up new opportunities for the synthesis and study of even higher acenes by selecting properly designed 
molecular hydroacene precursors and gives perspectives for fine tuning of their electronic and magnetic properties by atomically precise substitutional doping. Furthermore, the use of partially saturated acene precursors equipped with reactive functionalities may also allow for further onsurface processing, which could lead to more complex functional materials based on covalently linked acene substructures.

\section{Methods}

Sample preparation \& STM/AFM experiments. The experiments were performed in a ultrahigh vacuum multi-chamber system with the low-temperature scanning probe microscope that could operate in a scanning tunneling and non-contact atomic force microscopy mode. The microscope was manufactured by Omicron $\mathrm{GmbH}$. The $\mathrm{Au}(111)$ sample was prepared in a standard procedure of thermal annealing at $450{ }^{\circ} \mathrm{C}$ and subsequent $\mathrm{Ar}^{+}$ion bombardment. The molecules were evaporated thermally from a water-cooled Knudsen cell manufactured by Kentax $\mathrm{GmbH}$ on a sample kept at room temperature. The sublimation temperature was established at $260{ }^{\circ} \mathrm{C}$ with the application of a quartz microbalance giving a flux of approximately 0.05 monolayer/minute. After molecule deposition the sample was inserted into the microscope at liquid helium $(4.5 \mathrm{~K})$ temperature and subsequently measurements were performed. In all STM and STS experiments electrochemically etched Pt-Ir tips were applied as probes. All dI/dV maps and STS spectra were collected using lock-in amplifier (Zurich Instruments MFLI) with frequency of $610 \mathrm{~Hz}$ and amplitude of $23 \mathrm{mV}$ (rms). NC-AFM measurements were performed with the setup based on a qPlus sensor ${ }^{63}$ operated in frequency modulation mode with the bias voltage $\mathrm{V}$ set to $0 \mathrm{~V}$. To obtain higher spatial resolution in AFM measurements, carbon monoxide molecules were deposited onto the gold surface and then picked up by the AFM tip using procedure described by Gross et $a l^{42}$. Sample annealing leading to thermally driven dehydrogenation was performed in a preparation chamber using resistive heater. The samples were heated for 15 minutes at temperatures ranging from $150{ }^{\circ} \mathrm{C}$ up to $300{ }^{\circ} \mathrm{C}$. The temperature of the samples during annealing was controlled by the thermocouple.

DFT calculations. First-principles calculations of gas-phase nonacene molecules and their diand tetrahydroderivatives were performed using density functional theory (DFT) with the projector augmented waves $(\mathrm{PAW})^{64}$ and van der Waals corrected exchange-correlation functional (vdW-DF) available in VASP (Vienna ab-initio simulation package) ${ }^{65-67}$. The optPBE$\mathrm{vdW}$ implementation of the vdW-DF method was used in all the calculations ${ }^{68-70}$.

The plane wave basis set was restricted by an energy cutoff of $700 \mathrm{eV}$. Only the $\Gamma$ point was used in the Brillouin zone sampling. The total energy convergence criterion was chosen to be $10^{-6} \mathrm{eV}$. The atomic positions were fully relaxed by a conjugate gradient method until the maximum force in any direction was less than $0.01 \mathrm{eV} / \AA$. The local density of states maps have been calculated according to the Tersoff-Hamann approach in the constant-current mode ${ }^{57}$. 


\section{References}

1. Bendikov, M., Wudl, F.; Perepichka, D. F. Tetrathiafulvalenes, Oligoacenenes, and Their Buckminsterfullerene Derivatives: The Brick and Mortar of Organic Electronics. Chem. Rev. 104, 4891-4946 (2004).

2. Anthony, J. E. Functionalized Acenes and Heteroacenes for Organic Electronics. Chem. Rev. 106, 5028-5048 (2006).

3. Anthony, J. E. The Larger Acenes: Versatile Organic Semiconductors. Angew. Chem. Int. Ed. 47, 452-483 (2008).

4. Wang, C., Dong, H., Hu, W., Liu, Y., Zhu, D. Semiconducting $\pi$-Conjugated Systems in Field-Effect Transistors: A Material Odyssey of Organic Electronics. Chem. Rev. 112, 22082267 (2012).

5. Xiang, D., Wang, X., Jia, C., Lee, T., Guo, X. Molecular-Scale Electronics: From Concept to Function. Chem. Rev. 116, 4318-4440 (2016).

6. Sirringhaus, H. 25th Anniversary Article: Organic Field-Effect Transistors: The Path Beyond Amorphous Silicon. Adv. Mater. 26, 1319-1335 (2014).

7. Roncali, J., Leriche, P., Blanchard, P. Molecular Materials for Organic Photovoltaics: Small is Beautiful. Adv. Mater. 26, 3821-3838 (2014).

8. Kitamura, M., Imada, T., Arakawa, Y. Organic light-emitting diodes driven by pentacenebased thin-film transistors. Appl. Phys. Lett. 83, 3410 (2003).

9. Houk, K. N., Lee, P. S., Nendel, M. Polyacene and Cyclacene Geometries and Electronic Structures: Bond Equalization, Vanishing Band Gaps, and Triplet Ground States Contrast with Polyacetylene. J. Org. Chem. 66, 5517-5521 (2001).

10. Sun, Z., Ye, Q., Chi, C., Wu, J. Low band gap polycyclic hydrocarbons: from closed-shell near infrared dyes and semiconductors to open-shell radicals. Chem. Soc. Rev. 41, 7857-7889 (2012).

11. Brédas, J.-L., Beljonne, D., Coropceanu, V., Cornil, J. Charge-Transfer and Energy-Transfer Processes in $\pi$-Conjugated Oligomers and Polymers: A Molecular Picture. Chem. Rev. 104, 4971-5004 (2004).

12. Wiberg, K. B. Properties of Some Condensed Aromatic Systems. J. Org. Chem. 62, 57205727 (1997).

13. Son, Y.-W., Cohen, M. L. Louie, S. G. Half-metallic graphene nanoribbons. Nature 444, 347349 (2006).

14. Bursi, L., Calzolari, A., Corni, S., Molinari, E. Light-Induced Field Enhancement in Nanoscale Systems from First-Principles: The Case of Polyacenes. ACS Photonics 1, 10491058 (2014).

15. Dorel, R., Echavarren, A. M. Strategies for the Synthesis of Higher Acenes. Eur. J. Org. Chem. 14-24 (2017).

16. Shi, X., Chi, C. Different Strategies for the Stabilization of Acenes and Acene Analogues. Chem. Rec. 16, 1690-1700 (2016).

17. Thorley, K. J., Anthony, J. E. The Electronic Nature and Reactivity of the Larger Acenes. Isr. J. Chem. 54, 642-649 (2014).

18. Bettinger, H. F., Tönshoff, C. The Longest Acenes. Chem. Rec. 15, 364-369 (2015). 
19. Zade, S. S., Bendikov, M. Heptacene and Beyond: The Longest Characterized Acenes. Angew. Chem. Int. Ed. 49, 4012-4015 (2010).

20. Clar, E. Heptacen ein einfacher, „ultragrüner” Kohlenwasserstoff (Aromatische Kohlenwasserstoffe, XXXV. Mitteil.). Ber. Dtsch. Chem. Ges. 75B, 1330-1338 (1942).

21. Bailey, W. J., Liao, C.-W. Cyclic Dienes. XI. New Syntheses of Hexacene and Heptacene. $J$. Am. Chem. Soc. 77, 992-993 (1955).

22. Mondal, R., Adhikari, R. M., Shah, B. K., Neckers, D. C. Revisiting the Stability of Hexacenes. Org. Lett. 9, 2505-2508 (2007).

23. Mondal, R., Shah, B. K., Neckers, D. C. Photogeneration of Heptacene in a Polymer Matrix. J. Am. Chem. Soc. 128, 9612-9613 (2006).

24. Watanabe, M., Chang, Y. J., Liu, S.-W., Chao, T.-H., Goto, K., Islam, Md. M., Yuan, C.-H., Tao, Y.-T., Shinmyozu, T., Chow, T. J. The synthesis, crystal structure and charge-transport properties of hexacene. Nat. Chem. 4, 574-578 (2012).

25. Monahan N. R., Sun, D., Tamura, H., Williamns, K. W., Xu, B., Zhong, Y., Kumer, B., Nuckolls, C., Harutyunyan, A. R., Chen, G., Dai, H. - L., Beljonne, D., Rao, Y., Zhu, X. Y. Dynamics of the triplet-pair state reveals the likely coexistence of coherent and incoherent singlet fission in crystalline hexacene. Nat. Chem. 9, 341-346 (2017).

26. Einholz, R., Fang, T., Berger, R., Grüninger, P., Früh, A., Chassé, T., Fink, R. F., Bettinger, H. F. Heptacene: Characterization in Solution, in the Solid State, and in Films. J. Am. Chem. Soc. 139, 4435-4442 (2017).

27. Tönshoff, C., Bettinger, H. F. Photogeneration of Octacene and Nonacene. Angew. Chem. Int. Ed. 49, 4125-4128 (2010).

28. Kaur, I., Jazdzyk, M., Stein, N. N., Prusevich, P., Miller, G. P. Design, Synthesis, and Characterization of a Persistent Nonacene Derivative. J. Am. Chem. Soc. 132, 1261-1263 (2010).

29. Purushothaman, B., Bruzek, M., Parkin, S. R., Miller, A.-F., Anthony, J. E. Synthesis and Structural Characterization of Crystalline Nonacenes. Angew. Chem. Int. Ed. 50, 7013-7017 (2011).

30. Grill, L., Dyer, M., Lafferentz, L., Persson, M., Peters, M. V., Hecht, S. Nano-architectures by covalent assembly of molecular building blocks, Nat. Nanotech. 2, 687-691 (2007).

31. Cai, J., Ruffieux, P., Jaafar, R., Bieri, M., Braun, T., Blankenburg, S., Muoth, M., Seitsonen, A. P., Saleh, M., Feng, X., Müllen, K., Fasel, R. Atomically precise bottom-up fabrication of grapheme nanoribbons. Nature 466, 470-473 (2010).

32. Kolmer, M., Zebari, A. A. A., Prauzner-Bechcicki, J. S., Piskorz, W., Zasada, F., Godlewski, S., Such, B., Sojka, Z., Szymonski, M. Polymerization of Polyanthrylene on a Titanium Dioxide (011)-(2×1) Surface. Angew. Chem. Int. Ed. 52, 10300-10303 (2013).

33. Hla, S. -W. Atom-by-Atom Assembly. Rep. Prog. Phys. 77, 056502 (2014).

34. Hla S.-W., Bartels, L., Meyer, G., Rieder H.-K. Inducing all steps of a chemical reaction with the scanning tunnelling microscope tip: towards single molecule engineering, Phys. Rev. Lett. 85, 2777-2780 (2000).

35. Eigler D.M., Schweizer E.K. Positioning single atoms with a scanning tunnelling microscope. Nature 344, 524-526 (1990).

36. Repp, J., Meyer, G.; Paavilainen S., Olsson F.E., Persson M. Imaging bond formation beteen a gold atom and pentacene on an insulating surface. Science 312, 1196-1199 (2006).

37. Liljeroth P., Repp J., Meyer G. Current-induced hydrogen tautomerization and conductance switching of naphthalocyanine molecules. Science 317, 1203-1206 (2007). 
38. Pavliček, N., Schuler, B. Collazos, S., Moll, N., Perez, D., Guitian, E., Meyer, G., Peña, D., Gross, L. On-surface generation and imaging of arynes by atomic force microscopy. Nat. Chem. 7, 623-628 (2015).

39. Pavliček, N., Gross, L. Generation, manipulation and characterization of molecules by atomic force microscopy. Nat. Rev. Chem. 1, 0005 (2017).

40. Pavliček, N., Mistry, A., Majzik, Z., Moll, N., Meyer, G., Fox, D. J., Gross, L. Synthesis and characterization of triangulene. Nat. Nanotech. 12, 308-311 (2017).

41. Schuler, B., Fatayer, S., Mohn, F., Moll, N., Pavliček, N., Meyer, G., Peña, D., Gross, L. Reversible Bergman cyclization by atomic manipulation. Nat. Chem. 8, 220-224 (2016).

42. Gross, L., Mohn, F., Moll, N., Liljeroth, P., Meyer, G. The chemical structure of a molecule resolved by atomic force microscopy. Science 325, 1110-1114 (2009).

43. de Oteyza, D.G., Gorman, P., Chen, Y. -C., Wickenburg, S., Riss, A., Mowbray, D. J., Etkin, G., Pedramrazi, Z., Tsai, H. - Z., Rubio, A., Crommie, M. F. Direct imaging of covalent bond structure in single-molecule chemical reactions. Science 340, 1434-1437 (2013).

44. Albrecht, F., Pavliček, N., Herranz-Lancho, C., Ruben, M., Repp, J. Characterization of a surface reaction by means of atomic force microscopy. J. Am. Chem. Soc. 137, 7424-7428 (2015).

45. Kawai, S., Saito, S., Osumi, S., Yamaguchi, S., Foster, A. S., Spijker, P., Meyer, E. Atomically controlled substitutional boron-doping of graphene nanoribbons. Nat. Commun. 6, 8098 (2015).

46. Riss, A., Paz, A. P., Wickenburg, S., Tsai, H. -Z., De Oteyza, D. G., Bradley, A. J., Ugeda, M. M., Gorman, P., Jung, H. S., Crommie, M. F., Rubio, A., Fischer, F. R. Imaging singlemolecule reaction intermediates stabilized by surface dissipation and entropy. Nat. Chem. 8 , 678-683 (2016).

47. Repp, J., Mayer, G., Stojkovic, S. M., Gourdon, A., Joachim, C. Molecules on insulating films: scanning tunnelling microscopy imaging of individual molecular orbitals. Phys. Rev. Lett. 94, 026803 (2005).

48. Soe, W.-H., Manzano, C., De Sarkar, A., Chandrasekhar, N., Joachim, C. Direct Observation of molecular Orbitals of Pentacene Physisorbed on $\mathrm{Au}(111)$ by Scanning Tunneling Microscope. Phys. Rev. Lett. 102, 176102 (2009).

49. Godlewski, S., Kawai, H., Kolmer, M., Zuzak, R., Echavarren, A. M., Joachim, C., Szymonski, M., Saeys, M. Single-Molecule Rotational Switch on a Dangling Bond Dimer Bearing. ACS Nano 10, 8499-8507 (2016).

50. Godlewski, S., Kolmer, M., Engelund, M., Kawai, H., Zuzak, R., Garcia-Lekue, A., Saeys, M., Echavarren, A. M., Joachim, C., Sanchez-Portal, D., Szymonski, M. Interaction of a conjugated polyaromatic molecule with a single dangling bond quantum dot on a hydrogenated semiconductor. Phys. Chem. Chem. Phys. 18, 3854 (2016).

51. Krüger, J., Pavliček, N., Alonso, J. M., Pérez, D., Guitián, E., Lehmann, T., Cuniberti, G., Groudon, A., Meyer, G., Gross, L., Moresco, F., Peña, D. Tetracene Formation by On-Surface Reduction. ACS Nano 10, 4538-4542 (2016).

52. Krüger, J., Eisenhut, F., Alonso, J. M., Lehmann, T., Guitián, E., Pérez, D., Skidin, D., Gamaleja, F., Ryndyk, D. A., Joachim, C., Peña, D., Moresco, F., Cuniberti, G. Imaging the electronic structure of on-surface generated hexacene. Chem. Commun. 53, 1583-1586 (2017).

53. Athans, A. J., Briggs, J. B., Jia, W., Miller, G. P. Hydrogen-protected acenes. J. Mater. Chem. 17, 2636-2641 (2007). 
54. Dorel, R., McGonigal, P. R., Echavarren, A. M. Hydroacenes Made Easy by Gold(I) Catalysis. Angew. Chem. Int. Ed. 55, 11120-11123 (2016).

55. Bordwell, F. G., Cheng, J. P., Satish, A. V. \& Twyman, C. L. Acidities and hemolytic bond dissociation energies (BDEs) of benzyl-type carbon-hydrogen bonds in sterically congested substrates. J. Org. Chem. 57, 6542-6546 (1992).

56. van Scheppingen, W., Dorrestijn, E., Arends, I., Mulder, P. \& Korth, H.-G. Carbon-oxygen bond strength in diphenyl ether and phenyl vinyl ether: an experimental and computational study. J. Phys. Chem. A 101, 5404-5411 (1997).

57. Tersoff, J., Hamann, D. R. Theory and Application for the Scanning Tunneling Microscope. Phys. Rev. Lett. 50, 1998 (1983).

58. Hachmann, J., Dorando, J. J., Avils, M., Chan, G. K.-L. The radical character of the acenes: A density matrix renormalization group study. J. Chem. Phys. 127, 134309 (2007).

59. Bendikov, M., Duong, H. M., Starkey, K., Houk, K. N., Carter, E. A., Wudl, F. Oligoacenes: Theoretical Prediction of Open-Shell Singlet Diradical Ground States. J. Am. Chem. Soc. 126, 7416-7417 (2004).

60. Jiang, D., Dai, S. Electronic Ground State of Higher Acenes. J. Phys. Chem. A 112, 332-335 (2008).

61. Qu, Z. Zhang, D. Liu, C. Jiang, Y. Open-Shell Ground State of Polyacenes: A Valence Bond Study. J. Phys. Chem. A 113, 7909-7914 (2009).

62. Clar, E., John, Fr. Über eine neue Klasse tiefgefärbter radikalischer Kohlenwasserstoffe und über das vermeintliche Pentacen von E. Philippi; gleichzeitig Erwiderung auf Bemerkungen von Roland Scholl und Oskar Boettger. (Zur Kenntnis mehrkerniger aromatischer Kohlenwasserstoffe und ihrer Abkömmlinge, VII. Mitteil.). Ber. Drsch. Chem. Ges. 1930, 63, 2967-2977.

63. Giessibl, F. J., Atomic resolution on $\operatorname{Si}(111)-(7 \times 7) \operatorname{Si}(111)-(7 \times 7)$ by noncontact atomic force microscopy with a force sensor based on a quartz tuning fork. Appl. Phys. Lett. 76, 1470 (2000).

64. Blöchl, P.E. Projector augmented-wave method. Phys. Rev. B 50, 17953 (1994).

65. Dion, M., Rydberg, H., Schöder, E., Langreth, D. C., Lundqvist, B. I. Van der Waals Density Functional for General Geometries. Phys. Rev. Lett. 92, 246401 (2004).

66. Kresse, G., Furthmüller, J. Efficient iterative schemes for ab initio total-energy calculations using a plane-wave basis set. Phys. Rev. B 54, 11169 (1996).

67. Kresse, G. Joubert, D. From ultrasoft pseudopotentials to the projector augmented-wave method. Phys. Rev. B 59, 1758 (1999).

68. Roman-Perez, G., Soler, J. M. Efficient Implementation of a van der Waals Density Functional: Application to Double-Wall Carbon Nanotubes. Phys. Rev. Lett. 103, 096102 (2009).

69. Klimeš, J., Bowler, D. R., Michaelides, A. Chemical accuracy for the van der Waals density functional. J. Phys.: Condens. Matter 22, 022201 (2010).

70. Klimeš, J., Bowler, D. R., Michaelides, A. Van der Waals density functionals applied to solids. Phys. Rev. B 83, 195131 (2011).

\section{Acknowledgements}

The research was supported by the National Science Center, Poland (2014/15/D/ST3/02975) European Research Council (Advanced Grant No. 321066), MINECO/FEDER, UE (CTQ201675960-P), MINECO-Severo Ochoa Excellence Acreditation 2014-2018, SEV-2013-0319), and 
CERCA Program / Generalitat de Catalunya. MK acknowledges the support of the National Science Centre (Poland), project No. DEC-2014/15/B/ST5/04244. The STM experiments were carried out using equipment purchased with financial support from the European Regional Development Fund within the framework of the Polish Innovation Economy Operational Program (contract no. POIG.02.01.00-12-023/08). RZ acknowledges support received from KNOW (scholarship KNOW/59/SS/RZ/2016).

\section{Author contributions}

R.Z. performed all the STM/AFM experiments under the guidance of S.G., M.S., M.Ko. (STM/STS) and B.S. (AFM). R.D. synthesized the precursor molecules. M.Kr. performed the DFT calculations and image simulations. A.M.E initiated the work, oversaw and directed the synthesis of the acene precursors. S.G. supervised the research, conceived the experiments and prepared the interpretation of the STM/STS results. R.D. interpreted the isomerization of the intermediates. The manuscript was written by S.G. and R.D. All the authors discussed the results.

\section{Additional information}

Supplementary information is available in the online version of the paper. Reprints and permissions information is available online at www.nature.com/reprints. Correspondence and requests for materials should be addressed to S.G.

\section{Competing financial interests}

The authors declare no competing financial interests.

\section{Graphical abstract}

on-surface dehydrogenation

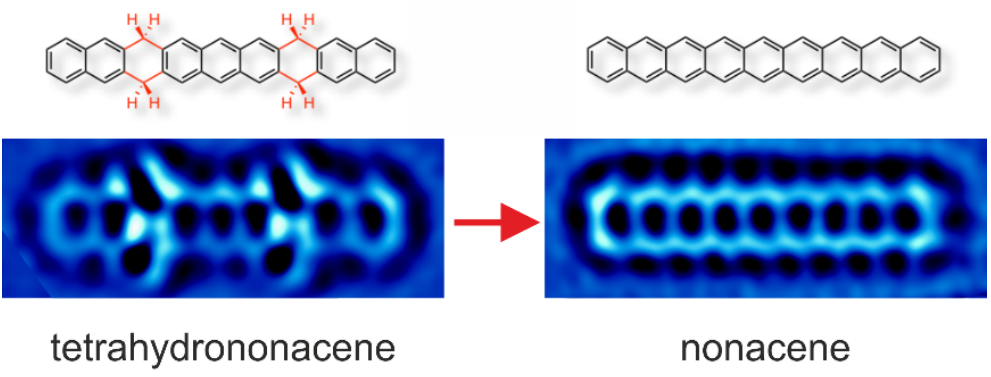

